PostgRAD. MED. J. (1965), 41, 37

\title{
FILARIASIS AS AN INCIDENTAL FINDING IN
}

\section{PREGNANCY}

\author{
Sidney Shaw, M.D., (Lond.), F.C.Path. \\ Geoffrey D. Pegrum, M.B., (Lond.), M.R.C.P., M.C.Path. \\ Department of Hamatology, Charing Cross Hospital Medical School, London, W.C.2.
}

THE IMPORTANCE of considering diseases, unknown or uncommon in this country, has received much attention recently owing to the increase in foreign travel and increased immigration. In particular, patients have been investigated for abnormal haemoglobins and alimentary parasites. Filariasis has received scant attention. Immigrants from Africa, India and the Far East may be infested by the nematode worms which give rise to the various forms of filariasis. At the time of leaving their native country patients may have few or no symptoms and as they move away from the insect vectors of the disease, spread in our community does not occur. Recently on routine blood examination two otherwise unsuspected cases of filariasis have come to our notice (Shaw and Pegrum, 1963).

\section{Case No. 1.}

Mrs. I's blood was taken for assay of Rhesus antibodies during pregnancy; the serum in which microfilaria were first seen was two days old (Fig. 1). The patient, a multigravida aged 24 , had been in England for about 2 years. She came from the Ijebu district of Western Nigeria. At the time the microfilaria were discovered she was 36 weeks pregnant. She had no symptoms whatsoever and appeared to be perfectly healthy.

\section{Case No. 2.}

Mrs. A's blood was examined for sickle cells and microfilaria were seen in the wet sealed preparation. The patient, a primagravida aged 21 , had been in England for a year. She came from Lagos in Western Nigeria, and was apparently well.

\section{Findings}

Peripheral and venous blood was taken from both of these patients and many microfilaria were discovered.

Venous and capillary blood was taken during the day and night into sequestrene tubes. Direct preparations were made from fresh blood and scanned with a $2 / 3$ objective. Lashing microfilaria are easily discovered in this way. To identify the type, blood smears were made from fresh specimens; both thick and thin films were made and stained with Leishman and Jenner Giemsa stain. We also examined concentrated samples, these were prepared by taking $0.05 \mathrm{ml}$. of capillary blood into $1 \mathrm{ml}$. of W.B.C. diluting fluid, centrifuging for 5 minutes at 1,500 r.p.m. and then making a film of the deposit.

In our first case many microfilaria were found in the peripheral and venous blood; in the second there were only a few in the peripheral blood and in the

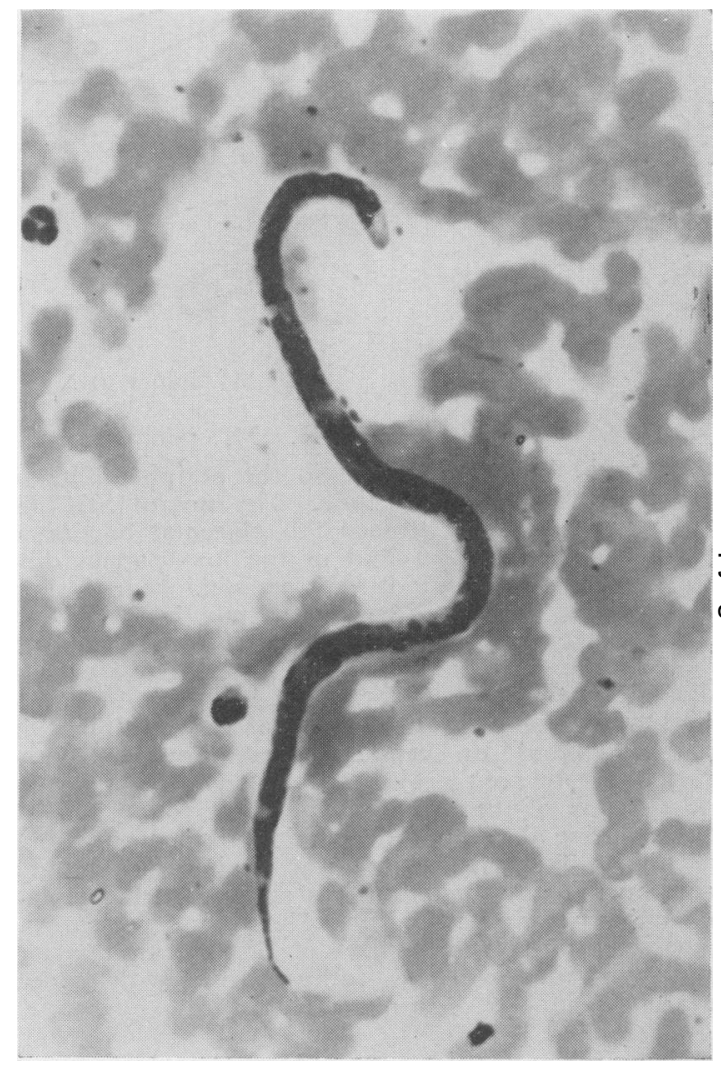

Fig. 1.

Mf. Loa in the peripheral blood from Case 1. (Sheath does not show up distinctly in this reproduction).

venous blood microfilaria were only present in the thick preparations. The first patient had an eosinophilia of $1,620 / \mathrm{cu}$. mm.; the second had no eosinophilia at first but later there were $1,200 / \mathrm{cu} . \mathrm{mm}$. eosinophils present. In both cases there was a mixed infection; the microfilaria appeared to be of the Loa Loa and Acanthocheilonema perstans types. This was decided by the specific morphology, including that of the posterior extremity where the column of nuclei reach to the tip and by the presence or absence of a sheath (Fig. 2).

Neither patients were anæmic and both continued their pregnancies uneventfully. Cord and maternal blood was taken from the patients at delivery. No microfilaria were found in the cord blood although 

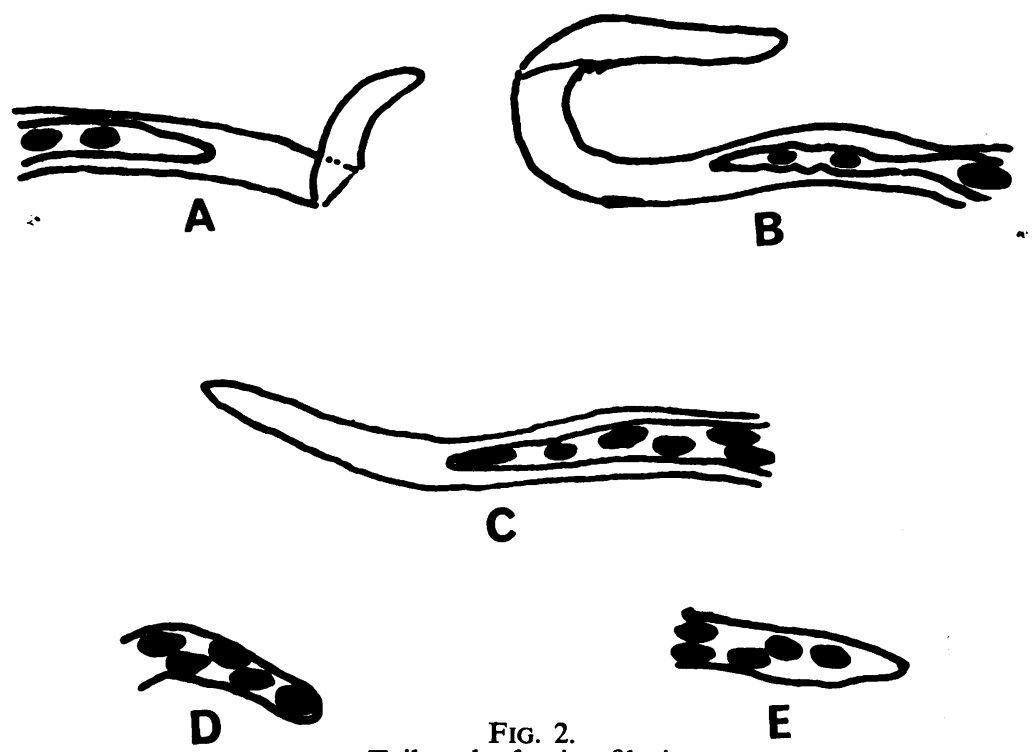

FIG. 2.

Tail end of microfilariæ.

A. Mf. bancrofti. C. Mf. Loa Loa.

D. Mf. perstans.

they could be demonstrated in the peripheral blood of the mothers at this time. The microfilaria did not appear to have crossed the placental barrier in these two cases. The child of the first patient died shortly after delivery with a congenital heart lesion; the child of the second was perfectly healthy.

In view of the heavy mixed infection it was decided to treat both patients after delivery with diethylcarbamazine-100 mg. t.d.s. for 14 days. Following treatment no microfilaria were found in the peripheral or venous blood, but when the patients were seen again after a few months scanty microfilaria of the Loa Loa type were found in both cases during the day and no microfilaria were found at night. The first patient was again pregnant and continued to be symptom-free and no further treatment has so far been given. The second patient has received a further course of diethylcarbamazine. Both patients will be followed up with repeat examinations for microfilaria.

\section{Discussion}

Filariasis usually presents with symptoms and signs referable to lymphatic obstruction by the adult worms or immature embryos. Loa Loa presents with 'Calabar' swellings or ocular symptoms. Not all the filarial worms are pathogenic. The adult worms of the superfamily Filarioidea, having microfilaria in the peripheral blood, are Wuchereria, Brugia, Loa, Acanthocheilonema and Mansonella. Those recognised as pathogens are Wuchereria bancrofti, Brugia malayi and Loa Loa.

Wuchereria bancrofti occurs in the tropics and subtropics, chiefly in Asia, Africa and Central America, but also in scattered coastal areas between the Mediterranean and Australia. The nematode worms of the filarial family are viviparous; they produce larvæ (the microfilaria)
B. Mf. malayi.

E. Mf. ozzardi.

which require a second host for their complete development. In the case of $W$. bancrofti this is a mosquito, usually Culex fatigans, but other mosquitos including Aedes and Anopheles may be the vector.

Brugia malayi is distributed in Indo-China? Malaya, Indonesia, India and Ceylon. The secondary host is again a mosquito. The microfilaria of both these types show a nocturnal periodicity.

Loa Loa infection, found in the central belt of Africa, has a diurnal periodicity and the secondary host is a biting fly of the chrysops genus.

The adult worms of Wuchereria and Brugia obstruct the lymphatic channels giving rise to Elephantiasis. In the case of Loa Loa 'Calabar' swellings may result from an inflammatory reaction caused by the presence of the adult worm in the subcutaneous tissues. The worm may be troublesome if it wanders across the eye.

Acanthocheilonema perstans occurs in Africa and South America and is spread by certain midges of the culicoides genus. Mansonella ozzardi is spread by a similar insect and is found in the West Indies and the north of South America. They are both non-pathogenic as far as is known but occasionally minor symptoms have been attributed to both. (Chandler and Read, 1961, Macfarlane, 1960). The microfilaria of both have no periodicity.

The diagnosis of filariasis is not usually difficult and can be made in the following ways:-

(a) the microfilaria can be found in the blood,

(b) a complement fixation test can be carried out,

(c) a skin sensitivity test can be performed. 
Filaria can be present for several years before symptoms develop, or they may be of a nonpathogenic type. In these cases the microfilaria may be found incidentally as in the present cases. The complement fixation test is positive in up to 50 per cent of the cases of filariasis but is negative in pure Acanthocheilonema perstans infection. This test is of value in confirming a diagnosis of tropical eosinophilia due to filariasis, the commonest cause of this condition. The skin sensitivity test is positive in 50 to $60 \%$ of all cases of filariasis. (Ahmed, 1960).

Microfilaria can persist in blood for up to 63 hours at $27-28^{\circ} \mathrm{C}$. (Tu and Hpay, 1962) and in stored blood at $4-6^{\circ} \mathrm{C}$. for as long as 24 days (Bird and Menon, 1961). There is therefore a reasonable chance of incidental discovery, particularly if the infestation is heavy. In all cases the type should be indentified by taking fresh specimens of peripheral blood during the day and night. Treatment in the case of the pathogenic varieties may prevent the development of symptoms. The treatment of the non-pathogenic varieties is a more difficult question. In the vector areas treatment is worthwhile to prevent the spread to others, but in this country it seems a little difficult to justify treatment unless there is a potentially pathogenic infection or the patient is returning to the vector area.

In the two cases described, in the absence of symptoms, it was decided to delay treatment until after delivery.

Treatment may be associated with gastrointestinal symptoms and allergic reactions. Anti- histamines and steroids have been used both to prevent and control these reactions. One course of therapy is not always curative as demonstrated in our cases and it is therefore important to examine for the presence of microfilaria after treatment.

\section{Summary}

Two patients are described in whom microfilaria were discovered during routine blood examinations.

The diagnosis and treatment of filariasis is discussed with particular reference to these two cases.

We would like to thank Dr. J. D. James of the North London Blood Transfusion Centre, who drew our attention to the first patient, and also Mr. K. Iles of the Photographic Department, Charing Cross Hospital Medical School, for the photographs.

\section{REFERENCES}

Ahmed, S. (1960): Filariasis, Brit. J. clin. Pract., 14, 697.

Bird, G. W. G., Menon, K. K. (1961): Survival of Microfilaria Bancrofti in Stored Blood, Lancet, ii, 721.

Chandler, A. C., Read, C. P. (1961): Introduction to parasitology; p. 488. New York: Wyllies.

Macfarlane, L. R. S. (1960): A Short Synopsis of Human Protozoology and Helminthology; p. 139, Edinburgh and London: E. \& S. Livingstone.

Shaw, S. and Pegrum, G. D. (1963): Filariasis in Pregnancy, Brit. med J., ii, 809.

TU, M., HPAY, Ma. (1962): Survival of Mammalian. Stages of Microfilariæ in vitro, Lancet, $\mathbf{i}, 166$.

\title{
TOXOPLASMOSIS PRESENTING AS A SWELLING IN THE AXILLARY TAIL OF THE BREAST
}

\author{
J. R. B. Turner, M.B., M.R.C.P. \\ Senior Registrar in General Medicine, United Leeds Hospitals.
}

OVER recent years cases of acquired toxoplasmosis have been recognised with increasing frequency. Serological studies suggest that between 25 and $50 \%$ of the population are infected with toxoplasma gondii at some time in their lives, but the infection is usually latent. Overt cases usually present as a lymphadenopathy with or without pyrexia, and this paper reports two cases of lymphadenopathy without pyrexia due to toxo. plasma in which the lymph gland enlargement was so close to the axillary tail of the breast as to appear as a breast swelling.

Case No. 1. An unmarried woman, aged 20, first attended at a Surgical Out-Patient Clinic in July, 1962 complaining of two swellings in the lateral aspect of the right breast, present for two weeks. She was otherwise symptom-free. Examination revealed two small, hard, non-tender swellings just lateral to the right breast. Her post-auricular lymph nodes were slightly enlarged and small nodes were palpable in the left carotid region and in the left axilla. The swellings near the breast were removed for histology.

Investigations. Chest X-ray normal. Hb. $100 \%$, WBC $4,800,53 \%$ polys., $41 \%$ lymphs., $6 \%$ monos. No atypical monocytes seen. E.S.R. (Westergren) 5 $\mathrm{mm}$./hr.

Histology. (Dr. W. Goldie) showed the biopsy specimens to be lymph nodes. The lymphoid follicles were hyperplastic with large Fleming centres and there was marked hyperplasia of the reticulum cells of the sinuses. The histological appearances were suggestive of toxoplasmosis. 